\title{
Suppressive Effect of Sinomenine Combined with 5-Fluorouracil on Colon Carcinoma Cell Growth
}

\author{
Ji-Xiang Zhang, Zi-Rong Yang, Dan-Dan Wu, Jia Song, Xu-Feng Guo, Jing \\ Wang, Wei-Guo Dong*
}

\begin{abstract}
It is reported that sinomenine (SIN) and 5-fluorouracil (5-FU) both are effective for colon cancer, but their cooperative suppressive effects and toxicity remain to be clarified in detail. This study aimed to determine suppressive effects and toxicity of sinomenine (SIN) plus 5-fluorouracil (5-FU) on LoVo colon carcinoma cells in vitro and in vivo. CCK-8, Hoechst 33258 staining and an annexin V-FITC/PI apoptosis kit were used to detect suppressive effects. Western blotting was applied to investigate the essential mechanism underlying SIN and 5-FU-induced apoptosis. SIN or 5-FU or both were injected into nude mice, and then suppressive effects and side effects were observed. SIN plus 5-FU apparently inhibited the proliferation of LoVo cells and induced apoptosis. Moreover the united effects were stronger than individually $(p<0.05)$. The results of annexin V-FITC /PI staining and Hoechst 33258 staining showed that the percentage of apoptotic cells induced by SIN and 5-FU combined or alone was significantly higher than the control group $(p<0.05)$. Expression of Bax and Bcl-2 was up-regulated and down-regulated respectively. SIN or 5-FU significantly inhibited effects on the volume of tumour xenografts and their combined suppressive effects were stronger $(p<0.05)$. No obvious side effects were observed. It was apparent that the united effects of SIN and 5-FU on the growth of colorectal carcinoma LoVo cells in vitro and in vivo were superior to those using them individually, and it did not markedly increase the side effects of chemotherapy.
\end{abstract}

Keywords: Colon carcinoma LoVo cells - chemotherapy - suppressive effects- sinomenine- 5-fluorouracil

Asian Pac J Cancer Prev, 15 (16), 6737-6743

\section{Introduction}

Colorectal cancer is the fourth most common cancer in the world, and significant international variations in the distribution of colorectal cancer have been observed (Center et al., 2009; Siegal et al., 2013). Meanwhile, colon cancer is more frequent than rectal cancer (Labianca et al., 2010; Di Lena et al., 2013). Recently, the main therapy on primary colon carcinoma is surgical resection, but only half of them can be cured with the rest hopeless. In addition, before the colon carcinoma was diagnosed successfully, the metastasis has already happened. For better prognosis after surgical resection or other clinical situations, chemotherapy as a relatively effective therapy is used more and more.

As the first-line chemotherapeutic agent to treat colon carcinoma, 5-fluorouracil (5-FU) has past 40 years (Beretta et al., 2004). Whereas the uncontrollable toxicity and dissatisfying effective rate which around $20-35 \%$ with median overall survival no more than 1 year could not meet the needs of treatment (Wu et al., 2013; Focan et al., 2013; Ghiringhelli et al., 2013). So to seek a way such as united therapy that can improve the effective rate and decrease toxicity of 5-FU on colon carcinoma is very important. Recently, several studies (Unal et al., 2013; Hakim et al., 2014) have discussed the combinational effect of 5-FU with other agents, they found that the combination chemotherapy of Gemcitabine, Folinic Acid and 5-FU has a more obvious anti-cancer effect but as well as toxicity. So the combination of a natural, low-toxicity and highefficacy agents with 5-FU may be better.

Sinomenine (SIN) is an alkaloid extracted from the Chinese medicinal plant, Sinomenium acutum, which has been utilized to treat rheumatoid arthritis (RA) in China for over 2000 years (Kok et al., 2005). The topical application of SIN in the treatment of rheumatoid arthritis has potential advantages (Zheng et al., 2007). It has previously been demonstrated that SIN exhibited a wide range of pharmacological actions including anti-inflammatory, anti-rheumatic, immunosuppressive, analgesic and antiarrhythmic effects (Shu et al., 2007; Teng et al., 2012; Song et al., 2013). And there are many other researches indicate that SIN has many pharmacological characters such as inducing apoptosis and anti-angiogenesis effect what has been treated as means of treating colon carcinoma (Kok et al., 2005; Ju et al., 2010; Chen et al., 2011). 
Moreover, that SIN could influence the expression of TNF (Huang et al., 2007; Chen et al., 2011; Yi et al., 2011) and other factors which may inhibit the growth and induce apoptosis has been proved. Just because of this, it was reported that SIN has an antitumor effect and low toxicity (He et al., 2005; Li et al., 2008).

In this research, we studied the suppressive effects and possible side effects of SIN combined with 5-fluorouracil on LKoVo colon carcinoma cells in both in vitro and in vivo models.

\section{Materials and Methods}

\section{Chemicals and cell culture}

$\mathrm{SIN}\left(\mathrm{C}_{19} \mathrm{H}_{23} \mathrm{NO}_{4} \cdot 0.3 \mathrm{CHC}_{13}, \mathrm{SIN}\right)$ was acquired from Sigma-Aldrich Co. LLC (Sigma365602, Shanghai, China) and was stored in a stock solution of $100 \mathrm{mM}$ in dimethyl sulfoxide (DMSO) at $-20^{\circ} \mathrm{C}$. 5-FU was acquired from Sigma-Aldrich Co. LLC (Sigma F6627, New York, USA). The colon carcinoma cell line LoVo was obtained from the China Center for Type Culture Collection (CCTCC). The cells were cultured in DMEM (Gibco BRL Gaithersburg, MD, USA), $10 \%$ fetal bovine serum (FBS, GibcoBRL Gaithersburg, MD, USA), $50 \mu \mathrm{g} / \mathrm{mL}$ streptomycin, $50 \mathrm{IU} /$ $\mathrm{ml}$ penicillin, and $2 \mathrm{mM}$ glutamine and then was incubated in a $5 \%$ humidified $\mathrm{CO}_{2}$ atmosphere at $37^{\circ} \mathrm{C}$.

\section{Cell growth suppressive effect studies in vitro}

Being used together or respectively, the suppressive effects of SIN and 5-FU on LoVo cells was evaluated by the measuring CCK-8 dye absorbance of living cells. After being seeded in 96 -well microtitre plates $\left(5 \times 10^{3}\right.$ cells per well) which was added by 5-FU (50, 100, 200, 400, 800 $\mu \mathrm{M}), \operatorname{SIN}(200,400,800,1600,3200 \mu \mathrm{M})$ or $5-\mathrm{FU}+\mathrm{SIN}$ after cultured in DMEM for 12 hours, LoVo cells were cultured for 48 hours at $37^{\circ} \mathrm{C}$. Then every well was added by $10 \mu \mathrm{LCCK}-8$ solution $(5 \mathrm{mg} / \mathrm{ml}$ in phosphate buffered saline (PBS)) and cultured for 2 hours at $37^{\circ} \mathrm{C}$. Then the optical density of each well at $450 \mathrm{~nm}$ was measured by a multiwell spectrophotometer reader (BIO-RAD iMark). It was triplicate for each assay.

\section{Cell apoptosis assay}

Hoechst 33258 was used to detect the morphological feature of apoptotic cells and was performed in strict accordance with the following procedure: The LoVo cells were cultured with DMEM for 12 hours after seeded in the germfree 6-well plates. Following the manufacturer's instructions (Beyotime, C0003), we fixed, washed and dyed the cells with Hoechst 33258 staining solution of $0.5 \mathrm{~mL}$ for 5 minutes at room temperature after exposure to SIN $(160 \mu \mathrm{M}), 5-\mathrm{FU}(160 \mu \mathrm{M})$ alone or combined for $48 \mathrm{~h}$. Then we used PBS to wash the cells twice and scored and categorized the stained nuclei according to the condensation and staining characteristics of chromatin. We chose ten random fields per dish to observe and count under a fluorescence microscope (OLYMPUS, Japan), and then expressed mean values as the percentage of apoptotic cells.

Annexin V-FITC kit was used to determine the percentage of cells which were at the apoptotic phase.
The plates seeded by LoVo cells were incubated by the culture medium with 5-FU or SIN or 5-FU+ SIN for 24 hours to 48 hours at $37^{\circ} \mathrm{C}$. After being washed twice with PBS of around $4^{\circ} \mathrm{C}$, the cells were resuspended in binding buffer to the concentration of $1 \times 10^{6}$ cells $/ \mathrm{ml}$. Then $100 \mu \mathrm{l}$ of the cell suspension, $5 \mu \mathrm{l}$ of Annexin V-FITC and $10 \mu \mathrm{l}$ of Propidium Iodide (PI) were mixed into a tube of $5 \mathrm{ml}$. After being centrifuged, the tube was incubated in dark for 15 minutes at room temperature. Then the cells which was added by $400 \mu$ of binding buffer were analysed quickly by flow cytometry.

\section{Western blot analysis}

Whole protein extracts were prepared and analyzed by western blotting using a wet transfer system (BioRad, Hercules, CA, USA). Protein samples (20 $\mu \mathrm{g})$ were resolved by electrophoresis of SDS-polyacrylamide gels and electro-transferred to nitrocellulose membranes. Transfer efficiency and homogeneous loading was assessed by Ponceau stain. Membranes were blocked for $1 \mathrm{~h}$ in $10 \%$ non-fat milk, incubated with titrated primary antibody over night at $4{ }^{\circ} \mathrm{C}$ and then labeled with the horseradish peroxidase conjugated secondary antibody for $2 \mathrm{~h}$ at ambient temperature. The following antibodies were used: Bax, Bcl-2, GAPDH (Santa Cruz Biotechnology, CA, USA) 1:1000. Antibody reaction was revealed by enhanced chemiluminescence (ECL) (Millipore, Bedford, MA, USA) and then exposed with Kodak X-ray film. Protein band intensity was determined using the CMIASWIN video imaging system (Bio-Rad, Hercules, CA, USA).

\section{Effect of sinomenine and 5-FU on tumour development in nude mice}

Thirty male BALB/c-nu/nu nude mice obtained from the Laboratory Animal Central of Wuhan University, 5-6 weeks old and about 16-18 g, were chosen to set up the model of tumour xenografts. These mice were fed by autoclaved water, germfree food filtered air and in the cages of an isolator unit that were autoclaved. Twenty-four of these mice were injected with $1 \times 10^{7}$ cells which were obtained after being disposed according to the process above. The rest 6 of these mice were injected with saline. The mice which had tumors of the similar sized were divided into four groups randomly and each group had 6 mice. The first group was given with saline; the second group was given with $5-\mathrm{FU} 12 \mathrm{mg} / \mathrm{kg} /$ day; the third group was given with SIN $25 \mathrm{mg} / \mathrm{kg} /$ day; and the fourth group was given $\mathrm{SIN}+5$-FU. After feeding of 3 weeks, the mice were executed to obtain tumors.

\section{HE staining and TUNEL assay}

After divided each tumor xenograft into two sections, we fixed one section in $4 \%$ formaldehyde, and remained another fresh. Having dehydrated with gradient ethanol, imbedded in paraffin, dewaxed and rehydrated with gradient ethanol in order, the above were respectively examined by histology both of HE staining and TUNEL technique. The treated specimens were observed and analyzed by using the upright fluorescence microscope (OLYMPUS-BX53, Japan). 


\section{Evaluation of side effects}

The side effects of SIN and 5-FU on nude mice were evaluated by the analysis of morphology (HE), bloodbiochemistry (AST, ALT, BUN and Cr) and blood cell.

\section{Statistical analysis}

All raw data were analysed with the SPSS software package, version 17.0 (SPSS Inc., Chicago, IL). When the $p$ value was less than 0.05 , differences were considered significant.

\section{Results}

The effects of SIN or 5-FU respectively on the growth of colon carcinoma LoVo cell

In vitro, the drugs significantly inhibited the effects of growth inhibition on LoVo cells with a dose-dependent manner $(p<0.01)$ (Figure 1). When the concentrations of SIN were $200 \mu \mathrm{M}$ and $3200 \mu \mathrm{M}$, the mean (SD) inhibition rate were 19.34 (1.39)\% and 61.53 (3.62)\%. Meanwhile,
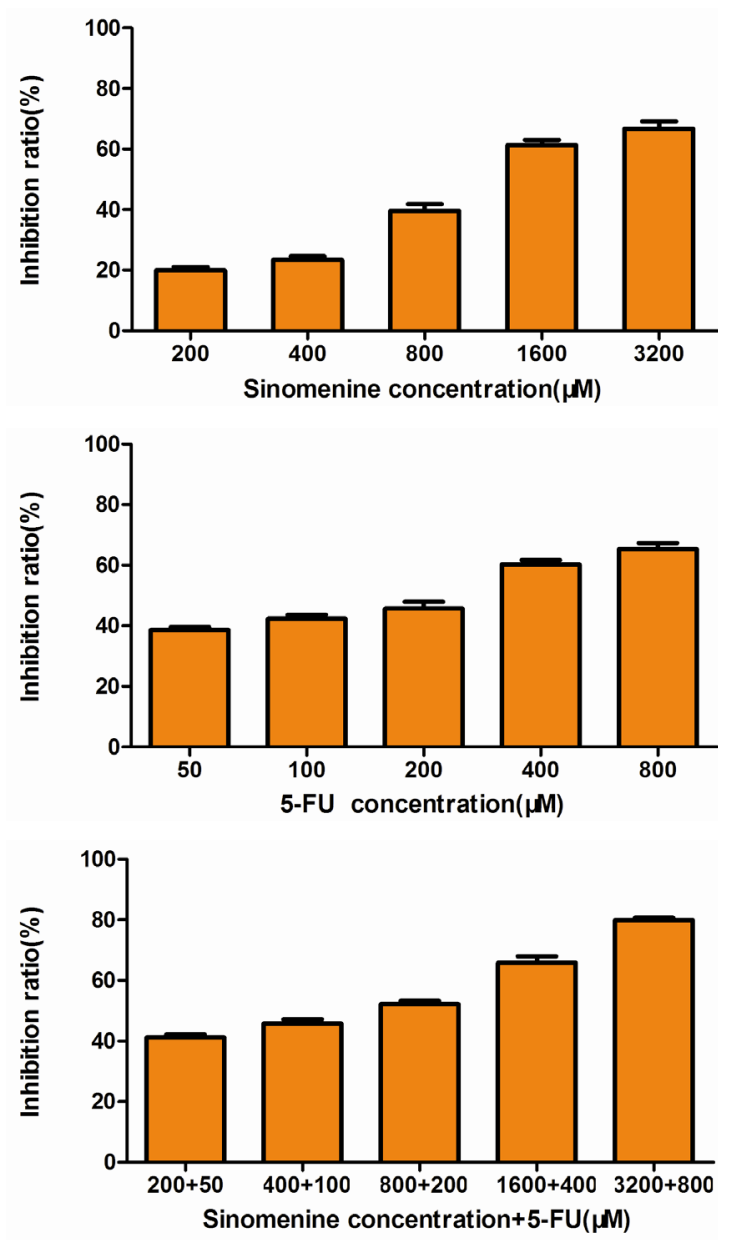

Figure 1. Effects of Sinomenine (SIN) or 5-fluorouracil (5-FU) Respectively on The Growth of Colon Carcinoma LoVo Cell. LoVo cells were incubated with SIN for $48 \mathrm{~h}$ at several concentrations $(200,400,600,800$, $1600,3200 \mathrm{M})$ and another $4 \mathrm{~h}$ with CCK-8. According to the optical density determined by spectrophotometer reader, the results were described as inhibition rate. There is a significant positive correlation between the inhibition rate and the dose of SIN. The results of 5-FU we obtained are similar to SIN. when the concentrations of 5-FU were at $50 \mu \mathrm{M}$ and 800 $\mu \mathrm{M}$, the rate were $38.99(0.71) \%$ and $61.78(0.88) \%$.

\section{Apoptosis induced by SIN or 5-FU}

As time goes on during the 48 hours of incubating, the proportion of Annexin V-positive/PI-negative cells were gradually increasing in the LoVo cells incubated at low concentrations of SIN $(200 \mu \mathrm{M})$ and 5-FU (50 $\mu \mathrm{M})$. Among the different groups, the effects of induced apoptosis of SIN groups and 5-FU groups were higher than the control groups $(p<0.01)$, and the $5-\mathrm{FU}+\mathrm{SIN}$ were higher than the SIN groups and 5-FU groups ( $p<0.01$, Figure 2).

Hoechst 33258 staining indicted that there were significant were detected in the number of apoptotic cells, and the percentage of apoptotic cells induced by SIN and 5-FU combined or alone were significant compared with the group control; furthermore, the combined apoptotic rate was more significant than the respective ones $(p<0.05$, Figure 3).

SIN and 5-FU induce apoptosis through the activation of the mitochondrial pathway

To further investigate the essential mechanism underlying SIN and 5-FU-induced apoptosis, their effects on the mitochondrial pathway were examined. SIN (1600 $\mu \mathrm{M})$ and 5-FU $(400 \mu \mathrm{M})$ treatment combined or alone caused an increase in Bax/GAPDH protein levels and a decrease in Bcl-2/GAPDH levels in LoVo cells, which led to a decrease in the antiapoptotic/proapoptotic (Bcl$2 /$ Bax) protein ratio (Figure 4 ). The apoptosis change in the combined group is significantly stronger than the respective ones.
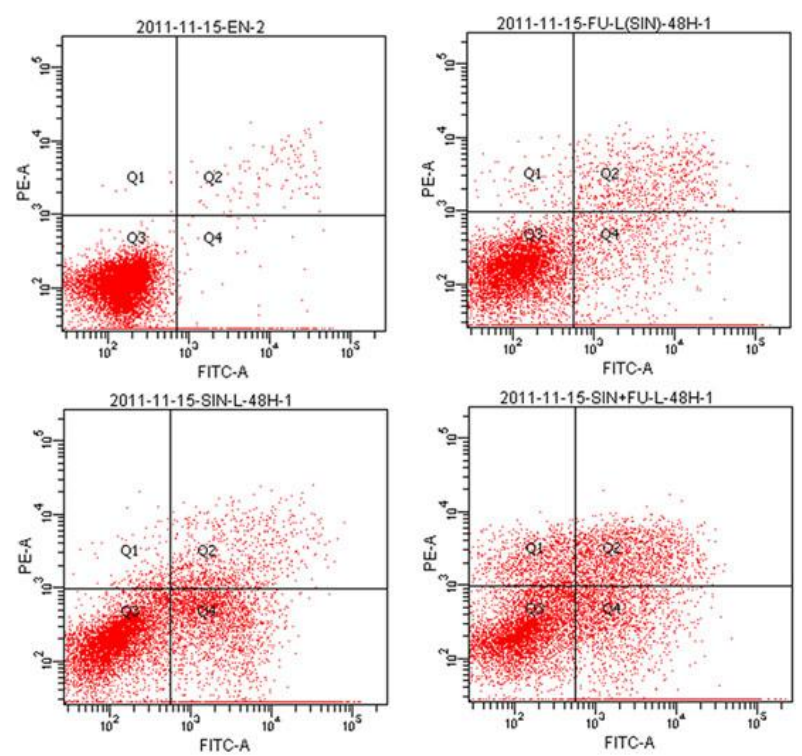

Figure 2. SIN and 5-FU Unitedly or Respectively Induced The Apoptosis of LoVo Cells. The apoptotic detection by Annexin V-FITC kit. We found that the groups of SIN and 5-FU unitedly or respectively can more significantly increase the rate of apoptosis than control group $(p<0.01)$, and united group more than respective groups $(p<0.01)$. 


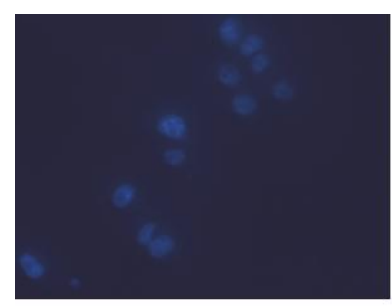

control

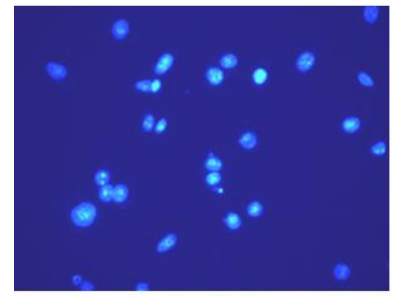

Sinomenine

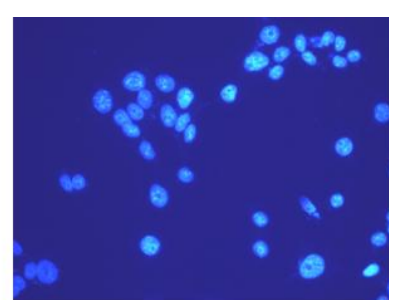

$5-F U$

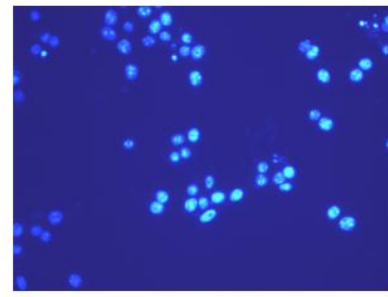

Sinomenine+5-FU
Figure 3. SIN and 5-FU Unitedly or Respectively Induced The Apoptosis of LoVo Cells. The apoptotic detection by Hoechst33258. The result reveals that the percentage of apoptotic cells of SIN+5-FU group was the highest, and then the SIN group and 5-FU group, and then the control group which had statistical significance $(p<0.01)$.

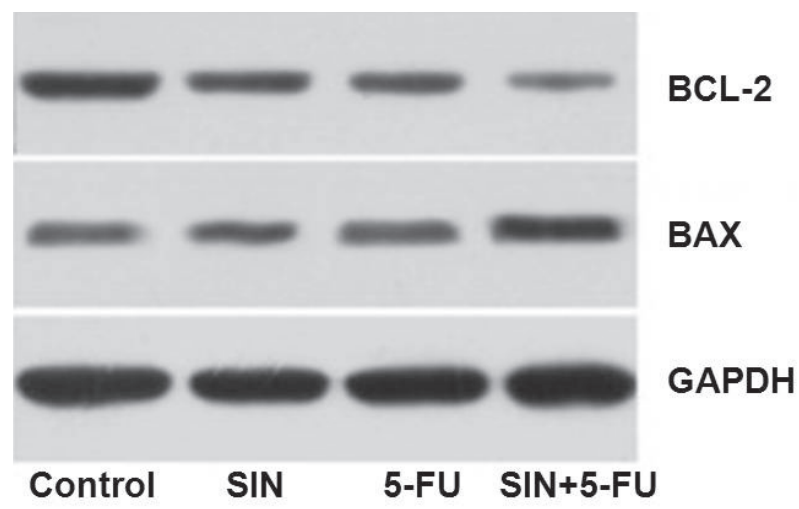

Figure 4. SIN and 5-FU Alone or Combined Induce Apoptosis in LoVo Cells through Mitochondrial Pathway. Western blot analysis results of Bcl-2 and Bax protein after SIN $(1600 \mu \mathrm{M})$ and 5-FU $(400 \mu \mathrm{M})$ treatment. GAPDH was used as a loading control.

Effects of SIN and 5-fluorouraci on tumor development in nude mice

All the 24 mice grew tumor of xenografts successfully without anyone died during the whole phase. After being fed for 14 days, the mean (SD) size of the tumor xenografts was $216.35 \mathrm{~mm}^{3}$. According to the results we got, SIN, 5-FU or SIN united with 5-FU all had significantly suppressive effects on the growth of LoVo cells. Compared with the control group, the volume and weight of tumor xenografts of the other 3 groups were all diminished significantly $(p<0.05$, Table 1 and Table 2 ) and the combination group's volume and weight were obviously less than SIN group and 5-fluorouraci group $(p<0.05$, Table 1 and Table 2$)$. In the control group, the size of the tumor xenografts was larger and larger, and some of them showed malignant such as dilated vessels and volcanoid ulcer. At the end of the study, the mean (SD)
Table 1. Inhibitory Effects of Sinomenine and 5-FU on LoVo Tumour Volume in Nude Mice

\begin{tabular}{llcc}
\hline Group & $\mathrm{n}$ & Volume $(\mathrm{mm} 3)$ & Inhibition rate $(\%)$ \\
\hline SIN & 6 & $171.81(31.90)^{* \#}$ & 66.30 \\
5-FU & 6 & $133.08(16.52)^{* \#}$ & 73.90 \\
SIN+5-FU & 6 & $50.69(14.41) \#$ & 90.06 \\
Control & 6 & $509.80(65.87)$ &
\end{tabular}

$* p<0.05$, significantly different from Sinomenine +5 -FU group; $\# p<0.05$, significantly different from control group

Table 2. Inhibitory Effects of Sinomenine and 5-FU on LoVo Tumour Weight in Nude Mice

\begin{tabular}{lllc}
\hline Group & $\mathrm{n}$ & Weight $(\mathrm{g})$ & Inhibition rate $(\%)$ \\
\hline SIN & 6 & $0.277(0.082)^{* \#}$ & 51.32 \\
5-FU & 6 & $0.240(0.061)^{* \#}$ & 57.77 \\
SIN+5-FU & 6 & $0.092(0.070) \#$ & 83.87 \\
Control & 6 & $0.568(0.125)$ & \\
\hline
\end{tabular}

$* p<0.05$, significantly different from Sinomenine +5 -FU group; $\# p<0.05$, significantly different from control group

size of tumor xenografts were respectively 171.81 (31.90), $133.08(16.52), 50.69(14.41), 509.80(65.87) \mathrm{mm}^{3}$ of the SIN group, 5-FU group, combination group and control group at the end of the experiment (Table1). The tumour inhibition rates of SIN group, 5-fluorouraci group or SIN united with 5-FU group was $66.30 \%, 73.90 \%$ and $90.06 \%$ (Table 1). The mean (SD) weight of tumor xenografts were respectively $0.277(0.082), 0.240(0.061), 0.092(0.070)$, $0.568(0.125) \mathrm{g}$ (Table2). And the inhibition rates of the three groups were $51.32 \%, 57.77 \%$ and $83.87 \%$ (Table 2). The results showed that suppressive effect of SIN united with 5-FU preceded the SIN or 5-FU in vivo.

It was showed by HE staining and TUNEL assay of the subcutaneous primary tumor sections that SIN and 5-FU used respectively or in combination induced significant apoptosis in vivo compared with the saline chloride tumor control group ( $p<0.05$, Figure 5), while the apoptotic degrees of each group significantly differed; the apoptotic rate of the combination group was higher than SIN group and 5-FU group. All the data above showed that the effects against colon carcinoma of SIN combined with 5-FU in vivo were superior to the effects of SIN and 5-FU used individually.

\section{Evaluation of side effects}

The 24 mice which were chosen and divided into 4 groups were necropsied to assess the side effects. In the 4 group, metastasis, peptic ulcer and haemorrhage, or injury of the liver and kidney all were not observed by the naked eye.

Among the SIN group, 5-FU group and SIN united with 5-FU group, there were no obviously difference of ALT and AST activities which was also not significantly raised (compared to the control group, $p<0.05$, Table 3 ). The size, weight and color of the liver and the renal of these groups was not significantly different and abnomal.

The result of blood cell count and the marrownucleated cell count showed that low SIN united with low 5-FU did not increase disadvantageously haematological changes. 
Table 3. Effect of Sinomenine Combined with 5-FU or Alone on Hepatic and Renal Function

\begin{tabular}{lccrr}
\hline Group & ALT $(\mathrm{U} / \mathrm{L})$ & AST $(\mathrm{U} / \mathrm{L})$ & Urea $(\mu \mathrm{mol} / \mathrm{L})$ & $\mathrm{Cr}(\mu \mathrm{mol} / \mathrm{L})$ \\
\hline SIN & $45.50(7.37)$ & $143.67(28.82)$ & $8.18(2.04)$ & $16.63(3.41)$ \\
5-FU & $45.33(6.02)$ & $145.33(18.53)$ & $8.04(1.20)$ & $15.73(4.30)$ \\
SIN+5-FU & $46.17(7.17)$ & $146.83(19.55)$ & $8.28(1.44)$ & $16.68(2.68)$ \\
Tumor control & $45.83(3.76)$ & $144.67(17.32)$ & $7.98(0.70)$ & $16.74(4.46)$ \\
Normal control & $46.00(5.00)$ & $145.17(11.27)$ & $8.09(1.03)$ & $16.29(4.51)$ \\
\hline
\end{tabular}

No differences were observed in the ALT, AST, Urea, and $\mathrm{Cr}$ among all groups $(p>0.05)$
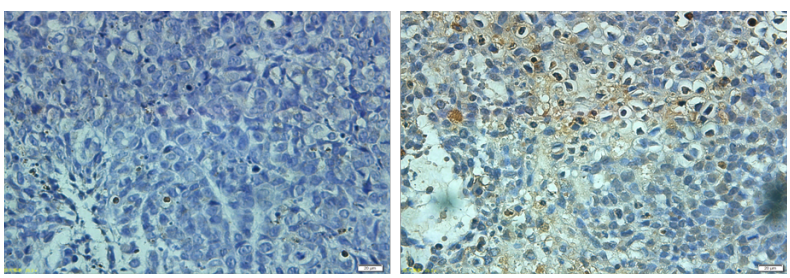

Control

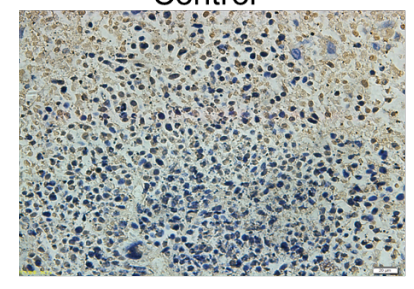

$5-\mathrm{FU}$

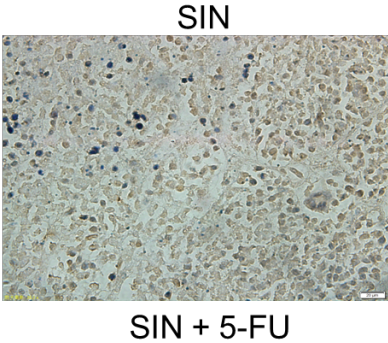

A

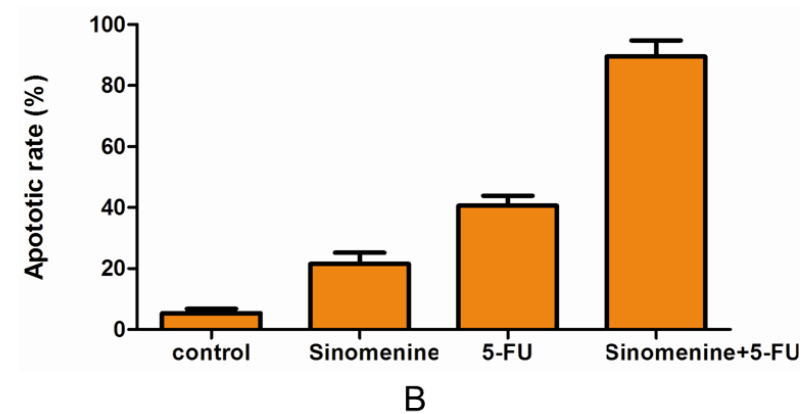

Figure 5. Detection of Apoptotic Cells in Xenograft Tumor Tissue by TUNEL Assay. SIN and 5-FU used individually or in combination induced significant apoptosis on LoVo cells in vivo compared with the saline chloride tumor control group $(P<0.05)$, while the apoptotic rate of the combination group was more significant than SIN group and 5-FU group.

\section{Discussion}

Widely in the world, the colon carcinoma is one of the malignant diseases which threaten people's life. Among all the cancers, the rate of estimated new cases in 2010, 2011 and 2012 was $6.72 \%, 6.34 \%, 6.29 \%$, and the rate of estimated deaths was $9.02 \%, 8.63 \%$, and $8.95 \%$ (Jemal et al., 2010; Jemal et al., 2011; Siegel et al., 2012). Because of the tremendous influencing on the public health, many treatments and early detections have been tried recently. Even through the advances in surgical techniques and radiotherapy being used, there were still approximately $50 \%$ of patients who were threatened by colon carcinoma newly diagnosed would die of their disease eventually, because of micrometastases. Thus chemotherapy has been treated as an important means to increase the survival rate of colon carcinoma patients.
With the effective rate around $10 \%$ and the median survival time of 9-11 months, 5-FU had acted as the first-line chemotherapeutic agent to treat colon carcinoma for over 40 years. And in the 1990s, chemotherapy was clearly demonstrated to successfully promote the effective rate and living quality of patients with metastatic colon carcinoma according to randomized comparisons. But what we could not ignore is the side effect which will weaken the curative effects. Furthermore in 1990s, the new drugs and combinations of chemotherapy had been investigated (Zhong et al., 2013).

For the past few years, more and more attentions were paid to the development of the curative effects of traditional medicines on the prevention and treatment of tumors. Extracted from the Chinese medicinal plant, Sinomenium acutum, SIN has been utilized to treat rheumatoid arthritis (RA) in China for over 2000 years. Previous reports have demonstrated a wide range of pharmacological actions including anti-inflammatory, anti-rheumatic, immunosuppressive, analgesic and antiarrhythmic effects (Shu et al., 2007; Teng et al., 2012; Song et al., 2013). But its potential value of anti-tumor by inhibiting proliferation and induced apoptosis was recognized in recent years (He et al., 2005; Li et al., 2008). Considering the value of anti-tumor and other pharmacological actions, we infer that the pair of SIN united with 5-fluorouracil can promote the anti-tumor effects and decrease the side effects.

In this research, we studied the suppressive effects of SIN united with 5-FU on colon carcinoma in vitro and in vivo and their side effects. We found that SIN united with 5-fluorouracil could inhibit proliferation. The united effects of SIN and 5-FU on the growth colorectal carcinoma LoVo cells in vitro and in vivo overmatched using them respectively. The study demonstrated that SIN united with 5-fluorouracil could induced apoptosis which was superior to that of SIN and 5-FU alone without obvious increasement of the side effects of chemotherapy.

Many reports show that SIN has the potent of inhibiting proliferation and inducing apoptosis on chondrocytes (Ju et al., 2010), CD4+ T cells (Shu et al., 2007), monocytes (Jiang et al., 2007) and tumor cells (Jiang et al., 2010). But the specific mechanism about how SIN inducing apoptosis has not been researched clearly. Apoptosis is a process of actively cellular death which is regulated by cells themselves. The signals for apoptosis can be initiated from outside the cell through the death-receptor pathway, or from inside the cell through the mitochondrial pathway (Chen et al., 2002; Green et al., 2005). The mitochondrial apoptotic pathway has been described as an important signaling pathway of apoptosis (Chen et al., 2002; Green et al., 2005). Anti- 
cancer agents extracted from Chinese herbs have been shown to induce the apoptosis of cancer cells through the mitochondrial apoptotic pathway (Magesh et al., 2009). 5-FU can inhibit the thymic pyrimidine nucleotidase of tumor cells and affects DNA stability (Yeh et al., 2000). It is found according to many studies that 5 -FU also induces apoptosis of gastroenteral carcinoma cells, including the attendance of p53, bcl-2, caspase- 3 and caspase- 8 (Ikebukuro et al., 2000; Wu et al., 2001; Matsuhashi et al., 2005; Baba et al., 2011). Mitochondria are thought to be a pivotal figure in apoptosis. In this study, we confirm that SIN combined with 5-FU can prompt apoptosis through the mitochondrial pathway.

What we could not escape is that SIN and 5-FU both have toxicity including gastroenteral disturbances, amenorrhoea, kidney dysfunction, leucopenia, thrombocytopenia and aplastic anaemia (Tang et al., 2007). Thus the model of tumor xenografts was designed in vivo and given different concentrations of SIN and 5-FU to investigate which concentration was the fewest toxic. Throughout the process of the study, there was no obvious side effect observed.

In conclusion, SIN united with 5-FU was superior to SIN and 5-FU respectively. Moreover the result that SIN united with 5-FU at lower concentrations will not increase the side effects encourages it is used as an effective antitumor method in clinical treatment.

\section{References}

Baba H, Hayashi N, Emi Y, et al (2011). A multicenter phase II clinical study of oxaliplatin, folinic acid, and 5-fluorouracil combination chemotherapy as first-line treatment for advanced colorectal cancer: a Japanese experience. Surg Today, 41, 1610-6.

Beretta GD, Milesi L, Pessi MA, et al (2004). Adjuvant treatment of colorectal cancer. Surg Oncol, 13, 63-73.

Center MM, Jemal A, Ward E (2009). International trends in colorectal cancer incidence rates. Cancer Epidemiol Biomarkers Prev, 18, 1688-94.

Chen DP, Wong CK, Leung PC, et al (2011). Anti-inflammatory activities of Chinese herbal medicine sinomenine and Liang Miao San on tumor necrosis factor-alpha-activated human fibroblast-like synoviocytes in rheumatoid arthritis. J Ethnopharmacol, 137, 457-68.

Chen M, Wang J (2002). Initiator caspases in apoptosis signaling pathways. Apoptosis, 7, 313-9.

Di Lena M, Travaglio E, Altomare DF (2013). New strategies for colorectal cancer screening. World J Gastroenterol, 19, 1855-60.

Focan C, Kreutz F, Graas MP, et al (2013). Phase I - II study to assess the feasibility and activity of the triple combination of 5-fluorouracil/folinic acid, carboplatin and irinotecan (CPT-11) administered by chronomodulated infusion for the treatment of advanced colorectal cancer. Final report of the BE-1603 study. Pathol Biol (Paris), 61, 27-31.

Ghiringhelli F, Bruchard M, Apetoh L (2013). Immune effects of 5-fluorouracil: Ambivalence matters. Oncoimmunology, 2, 23139.

Green DR (2005). Apoptotic pathways: ten minutes to dead. Cell, 121, 671-4.

Hakim L, Alias E, Makpol S, et al (2014). Gelam honey and ginger potentiate the anti cancer effect of 5-FU against HCT 116 colorectal cancer cells. Asian Pac J Cancer Prev,
15, 4651-7.

He X, Wang J, Guo Z, et al (2005). Requirement for ERK activation in sinomenine-induced apoptosis of macrophages. Immunol Lett, 98, 91-6.

Huang J, Lin Z, Luo M, et al (2007). Sinomenine suppresses TNF-alpha-induced VCAM-1 expression in human umbilical vein endothelial cells. J Ethnopharmacol, 114, 180-5.

Ikebukuro K, Adachi Y, Toki J, et al (2000). Morphological change, loss of deltapsi (m) and activation of caspases upon apoptosis of colorectal adenocarcinoma induced by $5-\mathrm{FU}$. Cancer Lett, 153, 101-8.

Jemal A, Bray F, Center MM, et al (2011). Global cancer statistics. CA Cancer J Clin, 61, 69-90.

Jemal A, Siegel R, Xu J, et al (2010). Cancer statistics, 2010. CA Cancer J Clin, 60, 277-300.

Jiang T, Zhou L, Zhang W, et al (2007) Sinomenine promotes differentiation but impedes maturation and co-stimulatory molecule expression of human monocyte-derived dendritic cells. Int Immunopharmacol, 7, 1102-10.

Jiang T, Zhou L, Zhang W, et al (2010). Effects of sinomenine on proliferation and apoptosis in human lung cancer cell line NCI-H460 in vitro. Mol Med Report, 3, 51-6.

Ju XD, Deng M, Ao YF, et al (2010). Protective effect of sinomenine on cartilage degradation and chondrocytes apoptosis. Yakugaku Zasshi, 130, 1053-60.

Kasibhatla S, Tseng B (2003). Why target apoptosis in cancer treatment? Mol Cancer Ther, 2, 573-80.

Kok TW, Yue PY, Mak NK, et al (2005). The anti-angiogenic effect of sinomenine. Angiogenesis, 8, 3-12.

Labianca R, Beretta GD, Kildani B, et al (2010). Colon cancer. Crit Rev Oncol Hematol, 74, 106-33.

Li L, Gao XL, Ding BX (2008). Gao and B.X. Ding, Inhibitory effect of sinomenine on $\mathrm{H} 2 \mathrm{O} 2$-induced apoptosis in neonatal rat cardiomyocytes. Zhongguo Zhong Yao Za Zhi, 33, 93941, 961 .

Magesh V, Lee JC, Ahn KS, et al (2009). Ocimum sanctum induces apoptosis in A549 lung cancer cells and suppresses the in vivo growth of Lewis lung carcinoma cells. Phytother Res, 23, 1385-91.

Matsuhashi N, Saio M, Matsuo A, et al (2005). The evaluation of gastric cancer sensitivity to 5-FU/CDDP in terms of induction of apoptosis: time- and p53 expressiondependency of anti-cancer drugs. Oncol Rep, 14, 609-15.

Shu L, Yin W, Zhang J, et al (2007). Sinomenine inhibits primary CD4+ T-cell proliferation via apoptosis. Cell Biol Int, 31, 784-9.

Siegal R, Naishadham D, Jemal A (2013). Cancer Statistics 2013. CA Cancer J Clin, 63, 11-30.

Siegel R, Naishadham D, Jemal A (2012). Cancer statistics, 2012. CA Cancer J Clin, 62, 10-29.

Song J, Bi H, Xie X, et al (2013). Preparation and evaluation of sinomenine hydrochloride in situ gel for uveitis treatment. Int Immunopharmacol, 17, 99-107.

Tang XY, Zhu YQ, Tao WH, et al (2007). Synergistic effect of triptolide combined with 5-fluorouracil on colon carcinoma. Postgrad Med J, 83, 338-43.

Teng P, Liu HL, Zhang L, et al (2012). Synthesis and biological evaluation of novel sinomenine derivatives as antiinflammatory agents. Eur J Med Chem, 50, 63-74.

Unal OU, Oztop I, Unek IT, et al (2013). Two-week combination chemotherapy with gemcitabine, high-dose folinic acid and 5 fluorouracil (GEMFUFOL) as first-line treatment of metastatic biliary tract cancers. Asian Pac J Cancer Prev, 14, 5263-7.

Waxman DJ, Schwartz PS, et al. (2003) Harnessing apoptosis for improved anticancer gene therapy. Cancer Res, 63, 8563-72.

Wu XX, Kakehi Y, Mizutani Y, et al (2001). Activation of 
caspase-3 in renal cell carcinoma cells by anthracyclines or 5-fluorouracil. Int J Oncol, 19, 19-24.

Wu Y, Wei ZW, He YL, et al (2013). Efficacy of adjuvant XELOX and FOLFOX6 chemotherapy after D2 dissection for gastric cancer. World J Gastroenterol, 19, 3309-15.

Yeh KH, Yeh SH, Hsu CH, et al (2000). Prolonged and enhanced suppression of thymidylate synthase by weekly $24-\mathrm{h}$ infusion of high-dose 5-fluorouracil. Br J Cancer, 83, 1510-5.

Yi H, Lu Y, Chen JK, et al (2010) Effect of sinomenine on tumor necrosis factor-alpha and nuclear factor-kappaB in the heterotopic tissue in rats with endometriosis. Nan Fang Yi Ке Dа Хие Хие Вао, 30, 1874-6.

Zheng H, Shi LF, Hu JH (2007). Assessment of in vitro and in vivo recovery of sinomenine using microdialysis. Skin Res Technol, 13, 323-9.

Zhong C, Liu H, Jiang L, et al (2013). Chemotherapy plus best supportive care versus best supportive care in patients with non-small cell lung cancer: a meta-analysis of randomized controlled trials. PLoS One, 8, e58466. 